\title{
TWO STEP SYNTHESIS OF INDAZOLE DERIVATIVES AND THEIR ANTI-CANCER EVALUATION
}

\author{
G. Laxman, Jagadeesh Kumar Ega and Kavitha Siddoju ${ }^{\bowtie}$ \\ Department of Chemistry, Chaitanya Deemed to be University, Kishanpura, Hanamkonda, \\ Warangal Urban 506001, Telangana State, India. \\ ${ }^{\otimes}$ Corresponding Author: siddojukavitha81@gmail.com
}

\begin{abstract}
Herein we described the synthesis of indazole derivatives through C-N cyclization and evaluated for their anticancer activities against three human cancer cell lines such as HeLa, MCF-7, and SKOV3 using MTT assay. The synthesized indazole hybrids are characterized based on 1H NMR, 13C NMR spectral data, and elemental analysis. The results indicated that the most potent molecule in this series is compound $3 \mathrm{~b}$ against MCF-7, compound $3 \mathrm{~h}$ against HeLa, and compound 3d against SKOV3, which showed the highest activity with an IC50 value of $32.92 \mu \mathrm{g} / \mathrm{ml}, 12.08 \mu \mathrm{g} / \mathrm{ml}$, and $29.06 \mu \mathrm{g} / \mathrm{ml}$ respectively.
\end{abstract}

Keywords: Indazole, Therapeutic Applications, Cyclization, Anticancer Activity

RASĀYAN J. Chem., Vol. 14, No.3, 2021

\section{INTRODUCTION}

Indazoles are heterocyclic moieties rarely found in nature. Indazole is described with a wide variety of medicinal activities. It is a great interest in the development of novel indazole-based therapeutic agents. The coming of single-iota catalysis as another boondocks that incorporates the benefits of both homogeneous and "heterogeneous catalysis"1-2 has gathered boundless consideration since its fundamental money in $2011^{3}$. Well-characterized active centers, stabilized by judiciously designed support, are highly desirable in heterogeneous catalysis for several reasons, their maximized atom utilization high surface coverage, and uniform structure to excellent selectivity and they have reactive sites wherein the isolated atoms and their local coordination environment can be reliably elucidated through spectroscopy techniques ${ }^{4}$. In the past years, these compounds have been well-established with their various functioning biological activities of antimicrobial ${ }^{5,6}$, antimalarial ${ }^{7}$, anti-inflammatory ${ }^{8}$, anticancer ${ }^{9,10}$ and antihyperlipidemic $^{11}$, antihyperglycemic ${ }^{12}$, cholesterol esterase inhibitor ${ }^{13}$, 15-hydroxyprostaglandin dehydrogenase(15-PGDH) inhibitor ${ }^{14}$, and bacterial arylamine N-Acetyltransferases (NATs) inhibitor ${ }^{15}$. Indazoles and their subsidiaries accomplished extraordinary importance for therapeutic physicists for the explanation that they have different natural exercises. Indazole is a fragrant heterocyclic natural compound found in particular normal items and medication particles. This indazole structure delineates a tremendous scope of natural and pharmacological activities ${ }^{16}$ comprehensive of antibacterial ${ }^{17}$, calming ${ }^{18}$, anticancer ${ }^{19}$, antitubercular. ${ }^{20}$ A portion of the indazole subordinates was portrayed as pharmacologically dynamic specialists as addressed in Figs.-1 to 3.

\section{EXPERIMENTAL}

\section{Synthesis of $\boldsymbol{E}$-hydrazone Esters from Diazo Compounds 2a-h}

Carboxylic ester $1(0.1 \mathrm{mmol})$, p-ABSA $(0.12 \mathrm{mmol})$, and $\mathrm{CH}_{3} \mathrm{CN}(0.3 \mathrm{ml})$ were added to a glass vial (4 $\mathrm{ml})$. With blending, 1, 5-diazabicyclo [4,3,0]non-5-ene (DBN) $(0.12 \mathrm{mmol})$ was added dropwise. The response combination was mixed at room temperature (RT) for 60 minutes. Then, at that point, $\mathrm{Pt} / \mathrm{Ce} 4+$ of $8.5 \mathrm{mg}$ (nano $\mathrm{CeO}_{2}$ ) impetuses and two $\mathrm{ml}$ of $\mathrm{CH}_{3} \mathrm{OH}$ were added straightforwardly and sonicated for $15 \mathrm{~min}$. From that point onward, $0.9 \mathrm{mmol}$ of smelling salts borane (Stomach muscle) was added. It was immediately shut, and the response blend was mixed for an additional 3 hours at RT. $0.1 \mathrm{mmol}$ of the 
RASĀYAN J. Chem.

Vol. 14 | No. 4 |2191-2195| October- December | 2021

diazo compound and $0.3 \mathrm{mmol}$ of abdominal muscle were added successively. $4 \mathrm{ml}$ was fixed rapidly, and the response combination was mixed at RT for $40 \mathrm{~min}$. besides conditions to the one-pot blend from aryl acetic acid derivation esters.<smiles>c1ccc2[nH]ncc2c1</smiles><smiles>c1ccc2n[nH]cc2c1</smiles><smiles>c1ccc2c(c1)CN=N2</smiles>

$1 H$-indazole

$2 \mathrm{H}$-indazole

$3 H$-indazole

Fig.-1: Structures of the indazole nucleus<smiles>Cc1ccc(Nc2nccc(Nc3ccc4c(C)n(C)nc4c3)n2)cc1S(N)(=O)=O</smiles><smiles>OCc1ccc(-c2nn(Cc3ccccc3)c3ccccc23)o1</smiles>

Pazopanib (I)

YC-1 (II)

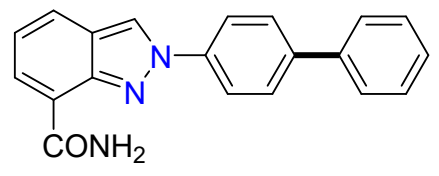<smiles>O=C(O)COc1nn(Cc2ccccc2)c2ccccc12</smiles>

Niraparib (III)

Bendec (IV)

Fig.-2: Some important indazole-containing drugs with pharmacological activity<smiles>O=C(O)c1nn(Cc2ccc(Cl)cc2Cl)c2ccccc12</smiles>

Lonidamine (V) (anticancer)<smiles>O=C(O)/C=C/c1nn(Cc2ccc(Cl)cc2Cl)c2cc(C(F)(F)F)ccc12</smiles>

Gamendazole (VI) (antifertility)

Fig.-3: Some medicinally potent indazoles

After the response, the blend was centrifuged to eliminate the impetus and washed multiple times with DCM. The supernatant was vapor under decreased pressing factor, and the residuals were exposed to be isolated utilizing a slight layer chromatography plate. The yield was determined by separating the measure of the got wanted product $\mathbf{2} \mathbf{a}-\mathbf{h}$ by the great yield. The gram-scale amalgamation of deformity rich $\mathrm{CeO}_{2}$ nanorods. ${ }^{21-22}$

\section{Synthesis of Indazole Derivatives}

$E$-hydrazone esters $\mathbf{2 a}$-h were treated with $10 \% \mathrm{CuI}, 0.9 \mathrm{mmol}$ of $\mathrm{Cs}_{2} \mathrm{CO}_{3}$ under DMSO solvent stirred for 2 hours and followed by $\mathrm{KOH}, \mathrm{EtOH}$ at $68{ }^{\circ} \mathrm{C}$ upon reflux condensation further hydrolysis indazole esters

(2)


RASĀYAN J. Chem.

Vol. 14 | No. 4 |2191-2195| October- December | 2021

derivatives are converted into indazole substituted carboxylic acids 3a-h. All the products are monitored by TLC and isolated by GC. All the derivatives were characterized by ${ }^{1} \mathrm{H}$ NMR, ${ }^{13} \mathrm{C}-\mathrm{NMR}$ spectra and CHN analyzer shown in Table-1 and 2.<smiles>[X]c1ccccc1/C(=N\N)OCC</smiles>

2a, $\mathrm{X}=\mathrm{H}, 88 \%$ yield<smiles>[X]c1ccccc1/C(=N\N)OCC</smiles>

2b, $\mathrm{X}=\mathrm{F}, 88 \%$ yield<smiles>[X]c1cc(Br)ccc1/C(=N\N)OCC</smiles>

2c, $\mathrm{X}=\mathrm{H}, 72 \%$ yield<smiles>[X]c1cc(C#N)ccc1/C(=N\N)OCC</smiles>

2d, $\mathrm{X}=\mathrm{Cl}, 70 \%$ yield<smiles>CCOC(=O)/C(=N/N)c1ccccc1</smiles>

$2 e, 5 \mathrm{~F}, 80 \%$ yield<smiles>[X]c1cc(C#N)ccc1/C(=N\N)C(=O)OCC</smiles>

2f, $\mathrm{X}=\mathrm{H}, 73 \%$ yield<smiles>[X]c1ccccc1/C(=N\N)C(=O)OCC</smiles>

2g, $\mathrm{X}=\mathrm{OH}, 83 \%$ yield<smiles>[X]c1cc(C(C)=O)ccc1/C(N)=N\N</smiles>

$2 \mathrm{~h}, \mathrm{X}=\mathrm{H}, 88 \%$ yield

Fig.-4: Yield Percentages of Compounds $\mathbf{2}$ a-h

\section{RESULTS AND DISCUSSION}

\section{Chemistry}

We have prepared a series of substituted indazole carboxylic acids 3a-h two steps (Scheme-1). In the first step, the one-pot union of $E$-hydrazone esters from carboxylic esters by treating with Carboxylic ester 1 $(0.1 \mathrm{mmol})$, p-ABSA $(0.12 \mathrm{mmol})$, and $\mathrm{CH}_{3} \mathrm{CN}(0.3 \mathrm{ml})$ were added to a glass vial $(4 \mathrm{ml})$. With blending, 1,5-diazabicyclo[4,3,0]non-5-ene $(\mathrm{DBN})(0.12 \mathrm{mmol})$ was added dropwise. The response blend was mixed at room temperature (RT) for 60 minutes. Then, $\mathrm{Pt} / \mathrm{Ce}^{4+}$ of $8.5 \mathrm{mg}$ (nano $\mathrm{CeO}_{2}$ ) catalysts and two $\mathrm{ml}$ of $\mathrm{CH}_{3} \mathrm{OH}$ were added directly and sonicated for $15 \mathrm{~min}$. $E$-hydrazone esters $\mathbf{2 a - h}$ were treated with $10 \% \mathrm{CuI}, 0.9 \mathrm{mmol}$ of $\mathrm{Cs}_{2} \mathrm{CO}_{3}$ under DMSO solvent stirred for 2 hours leads to cyclization and followed by $\mathrm{KOH}, \mathrm{EtOH}$ at $68{ }^{\circ} \mathrm{C}$ upon reflux condensation further hydrolysis indazole esters derivatives to result in the corresponding indazole substituted carboxylic acids $\mathbf{3 a} \mathbf{a} \mathbf{h}$ in quantitative yields.

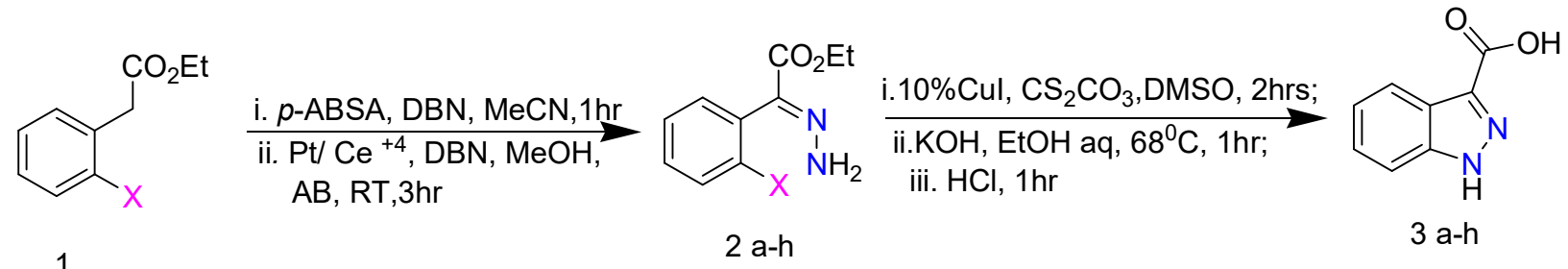

Scheme-1: Synthesis of Indazole derivatives 3(a-h)

(3a. yield $\%$ 71;3b. yield $\%$ 73; 3c. yield $\%$ 81; 3d. yield $\%$ 89; 3e. yield $\%$ 69; 3f. Yield $\%$ 79; 3g. yield $\%$ 80;

3h. yield\% 83.)

\section{Anti-Cancer Activity}

The orchestrated mixtures were evaluated for in vitro cytotoxic movement utilizing the microculture tetrazolium test, tried against human malignancy lines MCF-7, HeLa, and SKOV3. The different groupings of the engineered compounds (last focuses 1, 10,20,30,40, and $50 \mu \mathrm{g} / \mathrm{ml}$ ) were applied to ascertain IC50. The IC50 focus for each compound was determined concerning a control test, which addresses the fixation of those outcomes in a half reduction in cell development/expansion after $48 \mathrm{~h}$ hatching within the sight of the medication. The determined $\mathrm{IC}_{50}$ is arranged in Table-1. From Table 1 it is clear that compound $3 \mathrm{~b}$ against MCF-7, compound $3 \mathrm{i}$ against $\mathrm{HeLa}$, and compound $3 \mathrm{~d}$ against SKOV3, showed the most noteworthy action with an $\mathrm{IC}_{50}$ worth of $32.92 \mu \mathrm{g} / \mathrm{ml}, 12.08 \mu \mathrm{g} / \mathrm{ml}$, and $29.06 \mu \mathrm{g} / \mathrm{ml}$ individually. The cytotoxic exercises of combined mixtures were contrasted and the action showed by the reference drug Doxorubicin. 
RASĀYAN J. Chem.

Vol. 14 | No. 4 |2191-2195| October- December | 2021

Table-1: Anticancer Activity of Synthesized Compounds

\begin{tabular}{c|c|c|c}
\hline \multirow{2}{*}{ Entry } & \multicolumn{3}{|c}{ Anticancer Activity $\left(\mathrm{IC}_{50}\right.$ in $\left.\mu \mathrm{M}\right)$} \\
\cline { 2 - 4 } & MCF-7 & HeLa & SKOV3 \\
\hline $3 \mathrm{a}$ & 75.72 & 17.76 & 91.78 \\
\hline $3 \mathrm{~b}$ & 32.92 & 42.06 & $>100$ \\
\hline $3 \mathrm{c}$ & $>100$ & 33.87 & $>100$ \\
\hline $3 \mathrm{~d}$ & $>100$ & 57.05 & 29.06 \\
\hline $3 \mathrm{e}$ & $>100$ & 32.36 & $>100$ \\
\hline $3 \mathrm{f}$ & $>100$ & 62.77 & $>100$ \\
\hline $3 \mathrm{~g}$ & 35.15 & 22.07 & $>100$ \\
\hline $3 \mathrm{~h}$ & 91.66 & 12.08 & $>100$ \\
\hline Doxorubicin & 35.29 & 02.96 & 11.34 \\
\hline
\end{tabular}

${ }^{1} \mathrm{H},{ }^{13} \mathrm{C}-\mathrm{NMR}$ Spectra of $2 \mathrm{a}$

${ }^{1} \mathrm{H}$ NMR (400 MHz, Chloroform- $d$ ) $\delta[\mathrm{ppm}] 7.49$ (ddd, $\left.J=7.6,6.3,1.3 \mathrm{~Hz}, 2 \mathrm{H}\right), 7.45-7.40$ (m, 1H), 7.32 $-7.28(\mathrm{~m}, 2 \mathrm{H}), 6.27(\mathrm{~s}, 2 \mathrm{H}), 4.33(\mathrm{q}, J=7.1 \mathrm{~Hz}, 2 \mathrm{H}), 1.35(\mathrm{t}, J=7.1 \mathrm{~Hz}, 3 \mathrm{H}) ;{ }^{13} \mathrm{C}-\mathrm{NMR}(100 \mathrm{MHz}$, Chloroform- $d$ ) $\delta[\mathrm{ppm}] 164.4,137.4,129.6,129.3,129.2,128.8,61.3,14.3$.

${ }^{1} \mathrm{H},{ }^{13} \mathrm{C}-\mathrm{NMR}$ Spectra of $\mathbf{2 b}$

${ }^{1} \mathrm{H}$ NMR $(400 \mathrm{MHz}$, Acetonitrile- $d 3) \delta[\mathrm{ppm}] 7.55-7.48(\mathrm{~m}, 1 \mathrm{H}), 7.36-7.22(\mathrm{~m}, 3 \mathrm{H}), 6.78(\mathrm{~s}, 2 \mathrm{H}), 4.21$ $(\mathrm{q}, J=7.1 \mathrm{~Hz}, 2 \mathrm{H}), 1.27(\mathrm{t}, J=7.1 \mathrm{~Hz}, 3 \mathrm{H}) ;{ }^{13} \mathrm{C}-\mathrm{NMR}(100 \mathrm{MHz}$, Acetonitrile-d3) $\delta[\mathrm{ppm}] 164.7,160.3$ $(\mathrm{d}, J 1 \mathrm{~F}=244 \mathrm{~Hz}), 132.1(\mathrm{~d}, J 3 \mathrm{~F}=8 \mathrm{~Hz}), 131.6(\mathrm{~d}, J 3 \mathrm{~F}=4 \mathrm{~Hz}), 130.3,125.5(\mathrm{~d}, J 4 \mathrm{~F}=3 \mathrm{~Hz}), 118.9(\mathrm{~d}$, $J 2 \mathrm{~F}=18 \mathrm{~Hz}), 116.6(\mathrm{~d}, J 2 \mathrm{~F}=22 \mathrm{~Hz}), 61.1,14.2$.

\section{${ }^{1} \mathrm{H},{ }^{13} \mathrm{C}-\mathrm{NMR}$ Spectra of $2 \mathrm{c}$}

${ }^{1} \mathrm{H}$ NMR (400 MHz, Chloroform- $d$ ) $\delta[\mathrm{ppm}] 7.66-7.62(\mathrm{~m}, 2 \mathrm{H}), 7.22-7.19(\mathrm{~m}, 2 \mathrm{H}), 6.31$ (s, 2H), 4.31 (q, $J=7.1 \mathrm{~Hz}, 2 \mathrm{H}), 1.33(\mathrm{t}, J=7.1 \mathrm{~Hz}, 3 \mathrm{H}) ;{ }^{13} \mathrm{C}-\mathrm{NMR}(100 \mathrm{MHz}$, Chloroform- $d$ ) $\delta$ [ppm] 164.1, 136.1, $132.5,130.6,128.3,123.7,61.4,14.3$.

${ }^{1} \mathrm{H},{ }^{13} \mathrm{C}-\mathrm{NMR}$ Spectra of $2 \mathrm{~d}$

${ }^{1} \mathrm{H}$ NMR (400 MHz, Acetonitrile- $\left.d 3\right) \delta[\mathrm{ppm}] 8.02(\mathrm{~d}, J=8.5 \mathrm{~Hz}, 1 \mathrm{H}), 7.98-7.93(\mathrm{~m}, 2 \mathrm{H}), 7.82(\mathrm{~s}, 1 \mathrm{H})$, $7.63-7.56(\mathrm{~m}, 2 \mathrm{H}), 7.36(\mathrm{dt}, J=8.4,2.2 \mathrm{~Hz}$,

1H), $6.75(\mathrm{~s}, 2 \mathrm{H}), 4.26-4.20(\mathrm{~m}, 2 \mathrm{H}), 1.30-1.26(\mathrm{~m}, 3 \mathrm{H}) ;{ }^{13} \mathrm{C}-\mathrm{NMR}(100 \mathrm{MHz}$, Acetonitrile $d 3) \delta[\mathrm{ppm}]$ $165.3,135.8,133.9,133.8,129.2,129.1,128.7,128.3,128.3,127.4,127.1,127.0,61.0,14.3$.

\section{${ }^{1} \mathrm{H},{ }^{13} \mathrm{C}-\mathrm{NMR}$ Spectra of $2 \mathrm{e}$}

${ }^{1} \mathrm{H}$ NMR (400 MHz, Acetonitrile-d3) $\delta[\mathrm{ppm}] 7.27(\mathrm{~s}, 2 \mathrm{H}), 4.24(\mathrm{q}, J=7.1 \mathrm{~Hz}, 2 \mathrm{H}), 1.28(\mathrm{t}, J=7.1 \mathrm{~Hz}$, $3 \mathrm{H}) ;{ }^{13} \mathrm{C}-\mathrm{NMR}(100 \mathrm{MHz}$, Acetonitrile- $d 3) \delta$ [ppm] 163.1, $145.0(\mathrm{~m}), 143.1(\mathrm{~m}), 141.1(\mathrm{~m}), 139.1(\mathrm{~m})$, $137.1(\mathrm{~m}), 119.4,105.6(\mathrm{~m}), 61.0,13.5$.

\section{${ }^{1} \mathrm{H},{ }^{13} \mathrm{C}-\mathrm{NMR}$ Spectra of $2 \mathrm{f}$}

${ }^{1} \mathrm{H}$ NMR (400 MHz, Chloroform- $d$ ) $\delta[\mathrm{ppm}] 7.75-7.70(\mathrm{~m}, 2 \mathrm{H}), 7.42-7.36(\mathrm{~m}, 2 \mathrm{H}), 6.26(\mathrm{~s}, 2 \mathrm{H}), 4.24$ $(\mathrm{q}, J=7.1 \mathrm{~Hz}, 2 \mathrm{H}), 1.28(\mathrm{~d}, J=7.1 \mathrm{~Hz}, 3 \mathrm{H}) ;{ }^{13} \mathrm{C}-\mathrm{NMR}(100 \mathrm{MHz}$, Chloroform- $d) \delta[\mathrm{ppm}] 163.7,135.0$, $132.9,131.6,130.0,118.2,113.3,61.7,14.3$.

\section{${ }^{1} \mathrm{H},{ }^{13} \mathrm{C}-\mathrm{NMR}$ Spectra of $2 \mathrm{~g}$}

${ }^{1} \mathrm{H}$ NMR (400 MHz, Chloroform- $d$ ) $\delta[\mathrm{ppm}] 7.75(\mathrm{~d}, J=8.4 \mathrm{~Hz}, 1 \mathrm{H}), 7.40-7.38(\mathrm{~m}, 1 \mathrm{H}), 7.34(\mathrm{~s}, 1 \mathrm{H})$, $7.30-7.27(\mathrm{~m}, 1 \mathrm{H}), 6.23(\mathrm{~s}, 2 \mathrm{H}), 4.21(\mathrm{q}, J=7.1 \mathrm{~Hz}, 2 \mathrm{H}), 1.25(\mathrm{t}, J=7.1 \mathrm{~Hz}, 3 \mathrm{H}) ;{ }^{13} \mathrm{C}-\mathrm{NMR}(100 \mathrm{MHz}$, Chloroform- $d$ ) $\delta[\mathrm{ppm}] 163.6,146.9,145.5,131.0,129.8,128.4,127.7,123.3,61.2,14.2$.

\section{${ }^{1} \mathrm{H},{ }^{13} \mathrm{C}-\mathrm{NMR}$ Spectra of $\mathbf{2 h}$}

${ }^{1} \mathrm{H}$ NMR (400 MHz, Acetonitrile- $\left.d 3\right) \delta[\mathrm{ppm}] 8.10-8.07(\mathrm{~m}, 2 \mathrm{H}), 7.44-7.41(\mathrm{~m}, 2 \mathrm{H}), 6.72(\mathrm{~s}, 2 \mathrm{H}), 4.24$ (q, $J=7.1 \mathrm{~Hz}, 2 \mathrm{H}), 2.63(\mathrm{~s}, 3 \mathrm{H}), 1.27(\mathrm{t}, J=7.1 \mathrm{~Hz}, 3 \mathrm{H}) ;{ }^{13} \mathrm{C}-\mathrm{NMR}(100 \mathrm{MHz}$, Acetonitrile- $d 3) \delta$ [ppm] $197.7,164.3,137.3,135.6,134.3,129.4,128.8,60.5,26.1,13.6$. 
RASĀYAN J. Chem.

Vol. 14 | No. 4 |2191-2195| October- December | 2021

${ }^{13}$ C-NMR Spectra of Product $(100 \mathrm{MHz}$, Chloroform- $d$ ) $\delta$ [ppm]

3a. $160,142.4,141.3,126.4,120.7,120.4,113.5$.

3c. $160,144.6,141.3,124.4,122.6,120.6,113.3$.

3d. $160,143.1,141.3,127.3,121.6,115.8,113.3,109.8$.

3e. $160,141.3,138.2,135,129.3,127.8,125.5,111.6$.

3f. $160,142.3,139.2,134,128.3,125.8,124.5,108.6$.

3g. $160,141.3,138.2,135,129.3,127.6,125.3,111.6$.

3h. $160,142.3,141.3,134.7,127.4,120.3,120.2,99.8,29.3$.

Table-2: Analytical Data of Series of Compounds 2 and 3 by CHN Analyzer

\begin{tabular}{c|c|c|c}
\hline \multirow{2}{*}{ Entry } & Molecular & \multicolumn{2}{|c}{ Analytical Data of CHN } \\
\cline { 3 - 4 } & Formulae & Calcd (\%) & Found (\%) \\
\hline 2a & $\mathrm{C}_{10} \mathrm{H}_{12} \mathrm{~N}_{2} \mathrm{O}_{2}$ & $\mathrm{C}, 62.49 ; \mathrm{H}, 6.29 ; \mathrm{N}, 14.57$ & $\mathrm{C}, 62.62 ; \mathrm{H}, 6.38 ; \mathrm{N}, 14.60$ \\
\hline $2 \mathrm{~b}$ & $\mathrm{C}_{10} \mathrm{H}_{11} \mathrm{FN}_{2} \mathrm{O}_{2}$ & $\mathrm{C}, 57.14 ; \mathrm{H}, 5.27 ; \mathrm{N}, 13.33$ & $\mathrm{C}, 58.04 ; \mathrm{H}, 5.39 ; \mathrm{N}, 14.00$ \\
\hline 2c & $\mathrm{C}_{10} \mathrm{H}_{11} \mathrm{BrN}_{2} \mathrm{O}_{2}$ & $\mathrm{C}, 44.30 ; \mathrm{H}, 4.09 ; \mathrm{N}, 10.33$ & $\mathrm{C}, 44.64 ; \mathrm{H}, 4.29 ; \mathrm{N}, 11.00$ \\
\hline $2 \mathrm{~d}$ & $\mathrm{C}_{11} \mathrm{H}_{10} \mathrm{ClN}_{3} \mathrm{O}_{2}$ & $\mathrm{C}, 52.50 ; \mathrm{H}, 4.01 ; \mathrm{N}, 16.70$ & $\mathrm{C}, 54.50 ; \mathrm{H}, 4.81 ; \mathrm{N}, 16.90$ \\
\hline 2e & $\mathrm{C}_{10} \mathrm{H}_{7} \mathrm{~F}_{5} \mathrm{~N}_{2} \mathrm{O}_{2}$ & $\mathrm{C}, 42.57 ; \mathrm{H}, 2.50 ; \mathrm{N}, 9.93$ & $\mathrm{C}, 44.27 ; \mathrm{H}, 2.89 ; \mathrm{N}, 11.00$ \\
\hline 2f & $\mathrm{C}_{11} \mathrm{H}_{11} \mathrm{~N}_{3} \mathrm{O}_{2}$ & $\mathrm{C}, 60.82 ; \mathrm{H}, 5.10 ; \mathrm{N}, 19.34$ & $\mathrm{C}, 61.82 ; \mathrm{H}, 6.10 ; \mathrm{N}, 20.14$ \\
\hline 2g & $\mathrm{C}_{10} \mathrm{H}_{12} \mathrm{~N}_{2} \mathrm{O}_{3}$ & $\mathrm{C}, 57.68 ; \mathrm{H}, 5.81 ; \mathrm{N}, 13.45$ & $\mathrm{C}, 59.28 ; \mathrm{H}, 6.71 ; \mathrm{N}, 14.15$ \\
\hline 2h & $\mathrm{C}_{12} \mathrm{H}_{14} \mathrm{~N}_{2} \mathrm{O}_{3}$ & $\mathrm{C}, 61.53 ; \mathrm{H}, 6.02 ; \mathrm{N}, 11.96$ & $\mathrm{C}, 62.33 ; \mathrm{H}, 7.02 ; \mathrm{N}, 12.11$ \\
\hline $3 \mathrm{a}$ & $\mathrm{C}_{8} \mathrm{H}_{6} \mathrm{~N}_{2} \mathrm{O}_{2}$ & $\mathrm{C}, 59.26 ; \mathrm{H}, 3.73 ; \mathrm{N}, 17.28$ & $\mathrm{C}, 61.06 ; \mathrm{H}, 4.73 ; \mathrm{N}, 19.28$ \\
\hline $3 \mathrm{~b}$ & $\mathrm{C}_{8} \mathrm{H}_{5} \mathrm{~N}_{3} \mathrm{O}_{2}$ & $\mathrm{C}, 57.68 ; \mathrm{H}, 5.81 ; \mathrm{N}, 13.45$ & $\mathrm{C}, 59.28 ; \mathrm{H}, 6.71 ; \mathrm{N}, 14.15$ \\
\hline $3 \mathrm{c}$ & $\mathrm{C}_{8} \mathrm{H}_{5} \mathrm{BrN}_{2} \mathrm{O}_{2}$ & $\mathrm{C}, 39.86 ; \mathrm{H}, 2.09 ; \mathrm{N}, 11.62$ & $\mathrm{C}, 41.86 ; \mathrm{H}, 3.19 ; \mathrm{N}, 13.02$ \\
\hline $3 \mathrm{~d}$ & $\mathrm{C}_{17} \mathrm{H}_{14} \mathrm{~N}_{2} \mathrm{O}$ & $\mathrm{C}, 57.76 ; \mathrm{H}, 2.69 ; \mathrm{N}, 22.45$ & $\mathrm{C}, 59.16 ; \mathrm{H}, 3.69 ; \mathrm{N}, 23.65$ \\
\hline $3 \mathrm{e}$ & $\mathrm{C}_{8} \mathrm{H}_{2} \mathrm{~F}_{4} \mathrm{~N}_{2} \mathrm{O}_{2}$ & $\mathrm{C}, 41.04 ; \mathrm{H}, 0.86 ; \mathrm{N}, 11.97$ & $\mathrm{C}, 43.03 ; \mathrm{H}, 1.16 ; \mathrm{N}, 13.67$ \\
\hline $3 \mathrm{f}$ & $\mathrm{C}_{8} \mathrm{H}_{3} \mathrm{~F}_{3} \mathrm{~N}_{2} \mathrm{O}_{2}$ & $\mathrm{C}, 59.76 ; \mathrm{H}, 2.69 ; \mathrm{N}, 22.45$ & $\mathrm{C}, 59.16 ; \mathrm{H}, 3.69 ; \mathrm{N}, 23.65$ \\
\hline 3g & $\mathrm{C}_{10} \mathrm{H}_{7 \mathrm{~F}} \mathrm{~N}_{2} \mathrm{O}_{3}$ & $\mathrm{C}, 41.04 ; \mathrm{H}, 0.86 ; \mathrm{N}, 12.97$ & $\mathrm{C}, 43.03 ; \mathrm{H}, 1.16 ; \mathrm{N}, 13.67$ \\
\hline 3h & $\mathrm{C}_{10} \mathrm{H}_{8} \mathrm{~N}_{2} \mathrm{O}_{3}$ & $\mathrm{C}, 58.82 ; \mathrm{H}, 3.95 ; \mathrm{N}, 13.72$ & $\mathrm{C}, 59.92 ; \mathrm{H}, 4.55 ; \mathrm{N}, 15.12$ \\
\hline
\end{tabular}

\section{Biological Evaluation}

Cytotoxicity assay (MTT) was evaluated for all the synthesized compounds. Three different cancer cell lines viz., SKOV3 - Ovarian cancer, MCF7 - Breast cancer and HeLa Cervical cancer malignant growth was acquired from the ATCC (Bethesda, MD, USA) and kept up in DMEM enhanced with $10 \% \mathrm{FBS}, 2 \mathrm{mM}$ l-glutamine, $100 \mathrm{U} / \mathrm{ml}$ penicillin, and $100 \mu \mathrm{g} / \mathrm{ml}$ streptomycin at $37{ }^{\circ} \mathrm{C}$ in a $5 \% \mathrm{CO}_{2}$ hatchery. After cultivating of cells in 96 well culture plates, permitted to join appropriately. Test mixtures of various fixations going from 1 to $50 \mu \mathrm{M}$ were included sets of three and hatched for $24 \mathrm{hr}$. The cells were then hatched with MTT $(0.5 \mathrm{mg} / \mathrm{mL})$ for $3 \mathrm{hr}$ and to break up the insoluble formazan precious stones $100 \mu \mathrm{DMSO}$ was added to each well. At long last, the absorbance of the plates was estimated utilizing a Collaboration H1 multi-mode plate per user, USA. Doxorubicin was utilized as a positive control for the examination.

\section{ACKNOWLEDGEMENT}

Author ${ }^{1}$ is thankful to University Grant Commission, (NFPWD-2018-20-TEL-7777) in New Delhi for providing financial support in research. The authors wish to thank anonymous support given by Chaitanya deemed to be University, Hanamkonda, Warangal Urban, TS, for facilitating laboratory and for biological evaluation.

\section{CONCLUSION}

The present study described the two-step synthesis and anti-cancer activity of derived substituted indazoles. The compounds $3 \mathrm{~b}, 3 \mathrm{i}$ and $3 \mathrm{~d}$ are effective in anticancer activity.

\section{REFERENCES}

1. A. Q. Wang, J. Li, T. Zhang, Nature Review Chemistry, 2, 65(2018).

2. X. Cui, W. Li, P. Ryabchuk, K. Junge, M. Beller, Nature Catalysis, 1, 385(2018).

3. B. Qiao, A. Wang, X. Yang, L. F. Allard, Z. Jiang, Y. Cui, J. Liu, J. Li, T. Zhang, Nature Chemistry, 3, 634(2011).

4. X.-F. Yang, A. Wang, B. Qiao, J. Li, J. Liu, T. Zhang, Account of Chemical Research, 46, 1740(2013), https://doi.org/10.1021/ar300361m 
RASĀYAN J. Chem.

Vol. 14 | No. 4 |2191-2195| October- December | 2021

5. X. Jin, C.J. Zheng, M.X. Song, Y. Wu, L.P. Sun, Y.J. Li, H. R. Piao, European Journal of Medicinal Chemistry, 56,(2012), https://doi.org/10.1016/j.ejmech.2012.08.026

6. X.F. Liu, C.J. Zheng, L.P. Sun, X.K. Liu, H.R. Piao, European Journal of Medicinal Chemistry,46, 3469(2011), https://doi.org/10.1016/j.ejmech.2011.05.012

7. S. Naresh, M. Sharma, K. Srivastava, S. Rajakumar, S. K. Puri, J. K. Saxena, M.S. Prem, P.M.S. Chauhan, Bioorganic \& Medicinal Chemistry, 17, 6451(2009), https://doi.org/10.1016/j.bmc.2009.05.075

8. L. Ma, C. Xie, Y. Ma, J.Liu, M. Xiang, X. Ye, H. Zheng, L. Chen, A. Peng, , S. Yang, T. Chen, Journal of Medicinal Chemistry, 54(7), 2060(2011), https://doi.org/10.1021/jm1011534

9. H. Chen, Y.H. Fan, A. Natarajan, Y. Guo, J. Iyasere, F. Harbinski, L. Luus, J. A, Halperin, Bioorganic \& Medicinal Chemistry Letters, 14(21),5401(2004), https://doi.org/10.1016/j.bmcl.2004.08.017

10. R. Romagnoli, P. G. Baraldi, M. K. Salvador, M. E. Camacho, J. Balzarini, J. Bermejo, F. Estévez, $\begin{array}{lllll}\text { European of Journal } & \text { Medicinal }\end{array}$ https://doi.org/10.1016/j.ejmech.2013.02.030

11. A. K. Mohammed Iqbal, A. Y.Khan, M. B. Kalashetti, N. S. Belavagi, Y.D. Gong, I. M. Khazi, European Journal of Medicinal Chemistry, 53, 308(2012), https://doi.org/10.1016/j.ejmech.2012.04.015

12. B.B. Lohray, V. Bhushan, B.P. Rao, G.R. Madhavan, K. N. Murali, Rao, S. Subramaniam, Journal of Medicinal Chemistry,41(10), 1619(1998), https://doi.org/10.1021/jm970444e

13. S. Heng, W.Tieu, S. Hautmann, K. Kuan, D. S. Pedersen, M. Pietsch, A.D. Abell Bioorganic \& Medicinal Chemistry, 19(24), 7453(2011), https://doi.org/10.1016/j.bmc.2011.10.042

14. Y. Wu, H.H.Tai, H. Cho, Bioorganic \& Medicinal Chemistry, 18(4), 1428(2010), https://doi.org/10.1016/j.bmc.2010.01.016

15. E. W. Brooke, S. G. Davies, A. W. Mulvaney, M. Okada, F. Pompeo, E. Sim, Bioorganic \& Medicinal Chemistry Letters, 13(15), 2527(2003), https://doi.org/10.1016/S0960-894X(03)00484-0

16. A.W. Schmidt, K. R. Reddy, H.J. Knölker, Chemical Reviews, 112(6), 3193(2012), https://doi.org/10.1021/cr200447s

17. P.Y. Wang, H.S. Fang, W.B. Shao, J. Zhou, Z. Chen, B. A. Song, S. Yang, Bioorganic \& Medicinal Chemistry Letters, 27(18), 4294(2017), https://doi.org/10.1016/j.bmcl.2017.08.040

18. T. Surendiran, International Journal of Pharmtech Research, 8, 183(2015).

19. P. Sathiyachandran, P. Manogaran, V. N. Nesterov, V. V. Padma, K. Rajendra Prasad, European Journal of Medicinal Chemistry, 150, 851(2018), https://doi.org/10.1016/j.ejmech.2018.03.056

20. S. Goverdhan, S. K. Marvadi, P.Yogeeswari, D.Sriram, K. Srinivas, Bioorganic \& Medicinal Chemistry Letters, 28(9), 1610(2018), https://doi.org/10.1016/j.bmcl.2018.03.048

21. C. Liu, Z.Chen, Y. Huan, X. Zhao, Keyu Xie, J.Stephen, J. Ming, Science Advances, 5 ,(2019), https://doi.org/10.1126/sciadv.aay 1537

22. S. Biswas, F.Ben, S.Van, W. Marjo, B. Narendraprasad Reddy, E. Gwilherm, Angewandte Chemie International Edition, 160(2021), https://doi.org/10.1002/anie.202106716

[RJC-6362/2021] 